SECTION 4. Computer science, computer engineering and automation.

Gapon Nikolay Valeryevich

Student of department "Radio-electronic systems"

SRI "Digital signal processing and computer vision"

Don State Technical University

Voronin Viacheslav Vladimirovich

Ph.D., associate professor of Department. "Radio-electronic systems"

SRI "Digital signal processing and computer vision"

Don State Technical University

Sizaykin Roman Alekseyevich

Student of department "Radio-electronic systems"

SRI "Digital signal processing and computer vision"

Don State Technical University

Fisunov Alexander Vladimirovich

Student of department "Radio-electronic systems"

SRI "Digital signal processing and computer vision"

Don State Technical University

\title{
ALGORITHM OF TWO-DIMENSIONAL SIGNALS COMPRESSION BASED IMAGE RECONSTRUCTION METHODS
}

A new algorithm for image compression, which involves the removal of square blocks of pixels in an image of fixed size, followed by the remaining blocks encoded by JPEG. The decoder performs a decoding JPEG followed by reduction of the remote blocks. As a method of image reconstruction using reconstruction method based on texture analysis.

Keywords: compression, reconstruction, interpolation error of reconstruction.

\section{АЛГОРИТМ СЖАТИЯ ДВУМЕРНЫХ СИГНАЛОВ НА ОСНОВЕ МЕТОДОВ РЕКОНСТРУКЦИИ ИЗОБРАЖЕНИЙ}

Предлагается новый алгоритм к сжатию изображений, который заключается в удалении квадратных блоков пикселей на изображении фиксированного размера с последуюшим кодированием оставшихся блоков методом JPEG. Декодер выполняет прочедуру декодирования JPEG с последующим восстановлением удаленных блоков. В качестве метода восстановления изображений используется метод реконструкиии на основе текстурного анализа.

Ключевые слова: сжатие, восстановление, интерполяционные ошибки при реконструкиии.

В настоящее время системы обработки изображений находят применение во многих областях науки и техники. Полученная видеоинформация в большинстве случаев проходит многоэтапную процедуру обработки. Задача эффективного сжатия данных всегда была одной из самых актуальных, а с развитием компьютерной, цифровой техники и Интернет она приобрела еще большее значение.

Целью работы является разработка алгоритма сжатия двумерных сигналов на основе реконструкции статических изображений. 
Предлагается использовать новый подход к сжатию изображений, который заключается в предварительном удалении блоков на изображении с однородными участками с последующей их реконструкцией. Общая блок-схема основных этапов алгоритма представлена на рисунке 1 . Кодер включает в себя два блока: блок удаления однородных блоков и блок прямого дискретного преобразования; декодер состоит из блоков: блок обратного дискретного преобразования и блок реконструкции удаленных блоков.

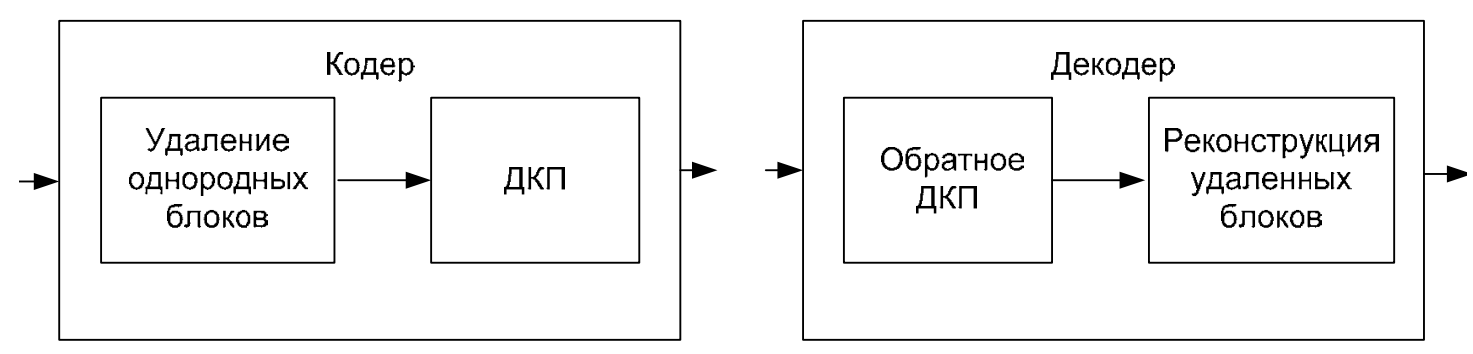

Рисунок 1 - Блок-схема основных этапов метода.

В кодере на первом шаге изображение разбивается на блоки размером 8 на 8 пикселей. Далее для каждого блока вычисляется мера контраста, которая используется как мера оценки однородности области. На втором шаге для изображения применяется сжатие с помощью ДКП преобразования.

В декодере применяется обратное ДКП преобразование. Далее изображение с удаленными блоками восстанавливается с помощью метода реконструкции изображений [1, с. 26-30].

Для удаления блоков используется процедура поиска квазистационарных блоков. Для чего используется описание текстуры с помощью моментов гистограммы интенсивностей элементов изображения. Пусть $L$ - случайная величина, которая определяет дискретную интенсивность изображения, $H(L(i, j))$ - соответствующие значения гистограммы. Известно, что $n$-й момент $L(i, j)$ относительно среднего значения определяется формулой:

$$
\mu_{n}(L)=\sum_{(i, j) \subset W}(L(i, j)-\bar{L})^{2} H(L(i, j))
$$

где $\bar{L}$ - среднее значение яркостей элементов локальной окрестности $W$.

Из выражения (1) следует, что $\mu_{0}=1$, а $\mu_{1}=0$. Второй момент, который называется дисперсией и обозначается как $\sigma^{2}(L)$, служит для описания текстуры. Он является также мерой контраста интенсивности и применяется для описания однородности поверхностей. В некоторых работах в качестве меры контраста текстуры предлагается использовать следующее выражение [2, с. 50-55]:

$$
C(i, j)=1-\frac{1}{1+k \sigma^{2}(L)}
$$

где $\sigma^{2}(L)$ - дисперсия в окрестности $n \times m, k=0,8$ - коэффициент нормирования.

После этапа удаления для остальных блоков применяется дискретное косинусное преобразование (ДКП). ДКП представляет изображение в виде суммы гармоник с различной амплитудой и частотой и является основой международного стандарта, который используется в алгоритме сжатия изображений с потерями JPEG.

В декодере для восстановления удаленных блоков предлагается использовать метод восстановления значений пикселей изображений на основе текстурного анализа. Данный метод заключается в поиске самоподобных областей на изображении и 
копировании их в область с отсутствующими пикселями. Данный метод позволяет корректно восстанавливать потерянные блоки, не размывая структуру изображения [3, C. 52-56.].

Далее приводятся некоторые примеры обработки изображений. Исследование эффективности проводятся на тестовых изображениях, где отсутствующие пиксели находятся на однородных участках текстурных областей.

На рисунке 2 представлен пример восстановления изображения «Лодка», а на рисунке 3 пример восстановления фрагмента изображения «Фруктыл» (а - исходное изображение; б - изображение с отсутствующими блоками пикселей; в - изображение, восстановленное методом реконструкции).

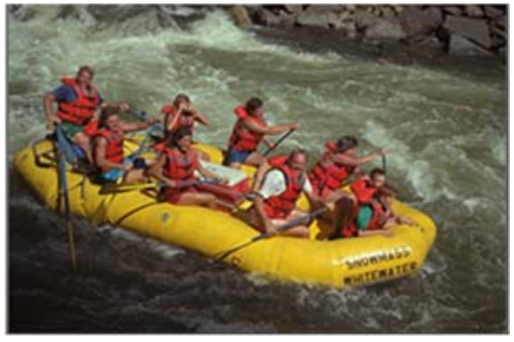

a)

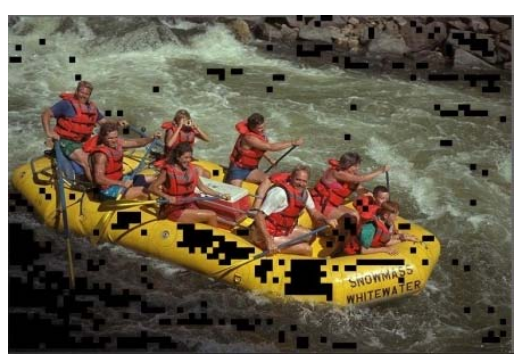

б)

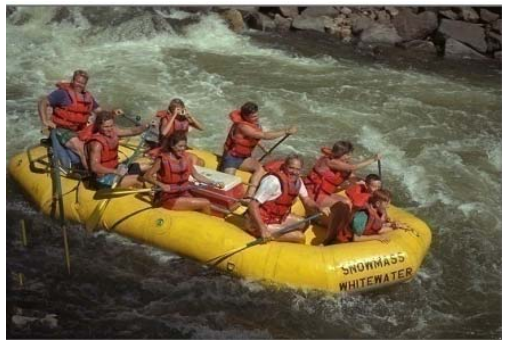

в)

Рисунок 2 - Восстановление изображения Лодка.

Анализ результатов обработки показывает, что предлагаемый метод позволяет синтезировать текстуру с одновременным восстановлением структуры изображения, при этом метод является робастным к размеру, форме, геометрическим особенностям области восстановления. Стоит отметить, что количество удаленных пикселей составляет примерно 30\% от их общего количества. Отсутствующие блоки находятся на участках со сложной текстурой, при этом предложенный метод не приводит к размытию текстуры при восстановлении больших областей с потерянными пикселями.

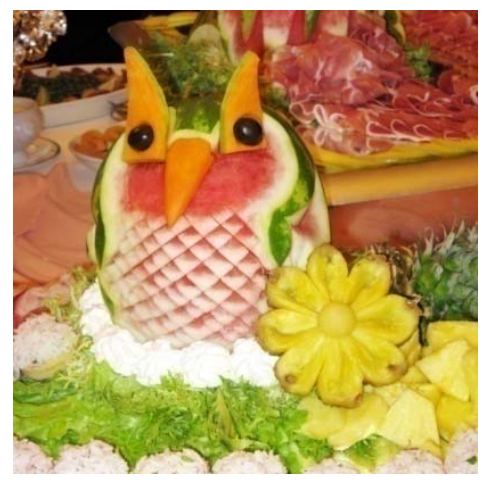

a)

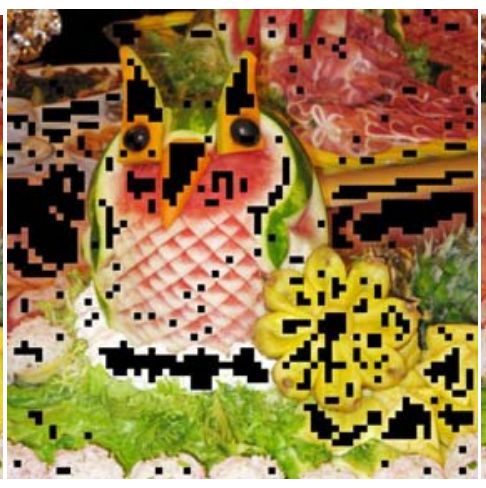

б)

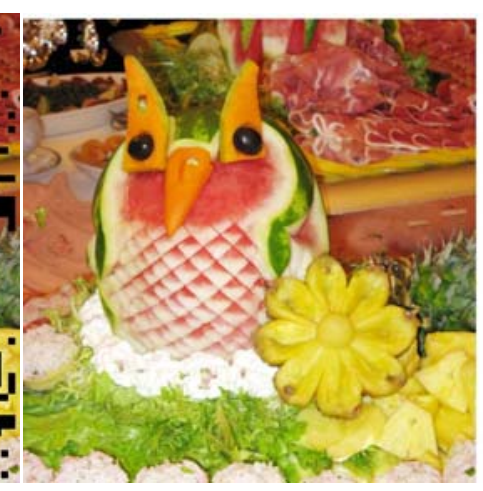

B)

Рисунок 3 - Восстановление фрагмента изображения Фрукты.

В заключении можно сделать следующие выводы.

Предлагается новый подход к сжатию изображений на основе метода реконструкции, который состоит из процедуры удаления квадратных блоков пикселей на изображении фиксированного размера с последующим кодированием оставшихся блоков методом JPEG. В качестве метода восстановления изображений выбран метод реконструкции на основе текстурного анализа. Данный метод заключается в поиске самоподобных областей на изображении и копировании их в область с отсутствующими пикселями. Сжатие в предлагаемом подходе происходит за счет уменьшения количества 
блоков изображения, для которых используется JPEG. Показана эффективность подхода на нескольких примерах при восстановлении блоков с пикселями на изображении.

\section{Список литературы}

1. Воронин В.В. Метод реконструкции изображений на основе интерполяции границ объектов кубическими сплайнами. Успехи современной радиоэлектроники, №6 Москва: Изд-во Радиотехника, 2012. - С. 26- 30.

2. Журавель И.М. Краткий курс теории обработки изображений / И.М. Журавель. M., 2002.

3. Марчук В.И., Воронин В.В. Реконструкция значений утраченных пикселей изображений в условиях ограниченной априорной информации//Научно-технические ведомости Санкт-Петербургского государственного политехнического университета. Информатика. Телекоммуникации. Управление. 2009. Т. 1. № 72. С. 52-56. 